Article

\title{
High Expression of KLF10 Is Associated with Favorable Survival in Patients with Oral Squamous Cell Carcinoma
}

\author{
Chung-Min Yeh ${ }^{1,2,+}$, Yi-Ju Lee ${ }^{3,4,5,+}$, Po-Yun Ko ${ }^{5,6}$, Yueh-Min Lin ${ }^{1,5, *, \ddagger}$ and Wen-Wei Sung ${ }^{5,7,8, *, \neq(1)}$ \\ 1 Department of Pathology, Changhua Christian Hospital, Changhua 50006, Taiwan; 28935@cch.org.tw \\ 2 Department of Medical Technology, Jen-Teh Junior College of Medicine, Nursing and Management, \\ Miaoli 35664, Taiwan \\ 3 Department of Pathology, Chung Shan Medical University, Taichung 40201, Taiwan; jasmine.lyl@gmail.com \\ 4 Department of Pathology, Chung Shan Medical University Hospital, Taichung 40201, Taiwan \\ 5 School of Medicine, Chung Shan Medical University, Taichung 40201, Taiwan; william70325@gmail.com \\ 6 Department of Medical Education, MacKay Memorial Hospital, Taipei 10491, Taiwan \\ 7 Institute of Medicine, Chung Shan Medical University, Taichung 40201, Taiwan \\ 8 Department of Urology, Chung Shan Medical University Hospital, Taichung 40201, Taiwan \\ * Correspondence: 93668@cch.org.tw (Y.-M.L.); flutewayne@gmail.com or sungww@csmu.edu.tw (W.-W.S.); \\ Tel.: +886-4-723-8595 (Y.-M.L.); +886-4-2473-9595 (ext. 10646) (W.-W.S.) \\ + Chung-Min Yeh and Yi-Ju Lee contributed equally in this work. \\ $\ddagger$ Yueh-Min Lin and Wen-Wei Sung contributed equally in this work.
}

Citation: Yeh, C.-M.; Lee, Y.-J.; Ko, P.-Y.; Lin, Y.-M.; Sung, W.-W. High Expression of KLF10 Is Associated with Favorable Survival in Patients with Oral Squamous Cell Carcinoma. Medicina 2021, 57, 17.

https://doi.org/10.3390/medicina 57010017

Received: 2 December 2020

Accepted: 23 December 2020

Published: 28 December 2020

Publisher's Note: MDPI stays neutral with regard to jurisdictional claims in published maps and institutional affiliations.

Copyright: () 2020 by the authors. Licensee MDPI, Basel, Switzerland. This article is an open access article distributed under the terms and conditions of the Creative Commons Attribution (CC BY) license (https: / / creativecommons.org/ licenses/by/4.0/).

\begin{abstract}
Background and Objectives: Krüppel-like transcription factor 10 (KLF10) plays a vital role in regulating cell proliferation, including the anti-proliferative process, activation of apoptosis, and differentiation control. KLF10 may also act as a protective factor against oral cancer. We studied the impact of KLF10 expression on the clinical outcomes of oral cancer patients to identify its role as a prognostic factor in oral cancer. Materials and Methods: KLF10 immunoreactivity was analyzed by immunohistochemical (IHC) stain analysis in 286 cancer specimens from primary oral cancer patients. The prognostic value of KLF10 on overall survival was determined by Kaplan-Meier analysis and the Cox proportional hazard model. Results: High KLF10 expression was significantly associated with male gender and betel quid chewing. The 5-year survival rate was greater for patients with high KLF10 expression than for those with low KLF10 expression (62.5\% vs. $51.3 \%$, respectively; $p=0.005$ ), and multivariate analyses showed that high KLF10 expression was the only independent factor correlated with greater overall patient survival. The significant correlation between high KLF10 expression and a higher 5-year survival rate was observed in certain subgroups of clinical parameters, including female gender, non-smokers, cancer stage T1, and cancer stage N0. Conclusions: KLF10 expression, detected by IHC staining, could be an independent prognostic marker for oral cancer patients.
\end{abstract}

Keywords: Krüppel-like factor 10; KLF10; prognosis; oral cancer; oral squamous cell carcinoma; overall survival

\section{Introduction}

Oral cancer is the sixth most common cancer in the world [1,2]. In spite of progress in research and therapy, the 5-year-survival rate has improved only minimally from $54 \%$ to $66 \%$ in the past 30 years $[3,4]$. Thus, prognostic tools that could promptly predict an unfavorable outcome of oral cancer are urgently needed for the early identification of potential outcomes and to modify existing treatment and follow-up strategies [5].

Carcinogenesis and tumor progression are believed to be substantially linked to the dysregulation of cell proliferation and the apoptosis resulting from cell mutation [6]. Krüppel-like-factor (KLF) family members are a group of transcriptional proteins containing three $\mathrm{C}_{2} \mathrm{H}_{2}$ zinc finger DNA-binding domains with a Krüppel linker structure between the zinc fingers. These proteins are involved in cell proliferation and the activation of 
apoptosis in normal tissues [7]. Krüppel-like transcription factor 10 (KLF10), also known as TIEG1, plays an important role in mediating the signaling of transforming growth factor beta (TGF $\beta$ ), a multifunctional cytokine with a sophisticated mechanism involving the expression of cell cycle regulators, cell proliferation, and activation of apoptosis [8,9]. KLF10 participates in multiple biological processes and diseases, including the anti-proliferative process and differentiation control [8-10]. Moreover, elevated intracellular levels of KLF10 tend to mimic the apoptotic and anti-proliferative effects of TGF $\beta$ [11-13]. The detailed mechanism of the complicated signaling cascade has been widely investigated in several cancers for the purpose of using KLF10 as a reliable prognostic index of cancer progression [14-19], and the significant prognostic value of KLF10 as a biomarker for predicting the survival of patients with pancreatic adenocarcinoma has been demonstrated in previous studies [7].

The remarkable role of KLF10 in mediating carcinogenesis has aroused interest in predicting the clinical outcome of oral cancer. In the present study, KLF10 levels were measured by the immunohistochemical (IHC) stain analysis of oral cancer specimens. Whether KLF10 protein expression is associated with specific clinical features and survival outcomes was also investigated in this study.

\section{Materials and Methods}

\subsection{Patients}

In this retrospective study, our study investigated tumor samples from patients with primary oral squamous cell carcinoma (OSCC). The cancers were staged according to the Cancer Staging Manual of the American Joint Committee on Cancer. The clinicopathological features collected included gender, age, risk factors, histological type, and TNM stage (tumor $(\mathrm{T})$, nodes $(\mathrm{N})$, and metastases $(\mathrm{M})$ satge) from the established database. The pathological diagnoses had been previously confirmed by two pathologists $[20,21]$. Those patients with missing clinical data or tissue loss during the IHC staining procedure were excluded from this study. The study was approved by the Institutional Review Board and Ethics Committee of the Changhua Christian Hospital, Changhua, Taiwan (IRB no. 131014) (3 March 2013). All methods were carried out in accordance with relevant guidelines and regulations.

\subsection{IHC Staining and Scoring of KLF10}

The IHC staining was performed at the Department of Surgical Pathology of Changhua Christian Hospital, Changhua, Taiwan, using the anti-human-KLF10 antibody (Santa Cruz, sc-23159; 1:75 dilution) as previously described [20,22]. The immunoreactivity scores were analyzed by the pathologists using scoring protocol as described previously [22,23], and the pathologists were blind to the clinical and prognostic data. A final consensus was obtained for each score by having all of the pathologists view the specimens simultaneously under a multi-headed microscope (Olympus BX51 10-headed microscope). The IHC staining scores were defined as cell staining intensity (0-3) multiplied by the percentage of stained cells $(0-100 \%)$, leading to scores from 0 to $300[20,22]$.

\subsection{Statistical Analyses}

The Student $t$ test, and the $\chi^{2}$ test were applied for continuous or discrete data analysis. The associations between KLF10 expression and overall survival were estimated using the Kaplan-Meier method and univariate analysis and assessed further using the logrank test [23,24]. Cox regression models of multivariate analysis were used to account for potential confounders with KLF10 expression fitted as an indicator variable. All the statistical analyses were conducted using SPSS statistical software (version 15.0; SPSS Inc., Chicago, IL, USA). All the statistical tests were two-sided, and values of $p<0.05$ were considered statistically significant. 


\section{Results}

\subsection{Patient Characteristics}

Overall, 286 patients $(241,84.3 \%$ male; $45,15.7 \%$ female) with a mean age of $56 \pm 11.5$ years (range: $31-90$ years) were enrolled in this retrospective study. The histological type of all the tumors collected was squamous cell carcinoma. Among the selected patients, several features were recorded and further categorized to observe the relationship between multiple factors and KLF10 expression. Overall, there were 117 (40.9\%) smokers and 169 (59.1\%) non-smokers. Fifty-eight $(20.0 \%)$ patients were positive for betel quid chewing versus $228(80.0 \%)$ patients who were negative. As for cancer stage, $56(19.6 \%)$ patients were in the early stage (I) and $230(80.4 \%)$ were in the late stages (II, III, IV). The overall 5 -year survival rate was $58.5 \%$, with a mean survival time of 4.7 years.

\subsection{Correlation between KLF10 Expression and Clinicopathological Features}

Representative images of the IHC staining of KLF10 are shown in Figure 1. The KLF10 expression score was $170 \pm 65$ (mean $\pm \mathrm{SD}$ ), and the median value was 90. Therefore, we defined a cytoplasmic KLF10 expression level $<90$ as a low expression. The relationships between KLF10 expression and multiple clinical parameters are listed in Table 1. Among all the parameters, male gender and betel quid chewing showed significant association with high KLF10 expression ( $p=0.015$ and $p=0.050$, respectively), but no significant association was observed between KLF10 and other parameters, such as age, smoking, tumor differentiation, stage, $\mathrm{T}$ value, or $\mathrm{N}$ value.

Table 1. The relationships between Krüppel-like transcription factor 10 (KLF10) expression and clinical parameters in oral cancer patients.

\begin{tabular}{|c|c|c|c|c|}
\hline \multirow[b]{2}{*}{ Parameters } & \multicolumn{4}{|c|}{ KLF10 Expression } \\
\hline & Case Number & Low & High & $p$ Value \\
\hline Age (years) & & $55.7 \pm 11.3$ & $56.7 \pm 11.5$ & 0.519 \\
\hline \multicolumn{5}{|l|}{ Gender } \\
\hline Female & 45 (15.7) & $18(40.0)$ & $27(60.0)$ & 0.015 \\
\hline Male & $241(84.3)$ & $55(22.8)$ & $186(77.2)$ & \\
\hline \multicolumn{5}{|l|}{ Smoking } \\
\hline No & $169(59.1)$ & $47(27.8)$ & $122(72.2)$ & 0.287 \\
\hline Yes & $117(40.9)$ & $26(22.2)$ & $91(77.8)$ & \\
\hline \multicolumn{5}{|l|}{ Betel quid chewing } \\
\hline No & $228(79.7)$ & $64(28.1)$ & 164 (71.9) & 0.050 \\
\hline Yes & $58(20.3)$ & $9(15.5)$ & $49(84.5)$ & \\
\hline \multicolumn{5}{|l|}{ Differentiation } \\
\hline Good & $45(15.7)$ & $11(24.4)$ & $34(75.6)$ & 0.856 \\
\hline Moderate + Poor & $241(84.3)$ & $62(25.7)$ & $179(74.3)$ & \\
\hline \multicolumn{5}{|l|}{ Stage } \\
\hline I & $56(19.6)$ & $15(26.8)$ & $41(73.2)$ & 0.809 \\
\hline $\mathrm{II}+\mathrm{III}+\mathrm{IV}$ & $230(80.4)$ & $58(25.2)$ & $172(74.8)$ & \\
\hline \multicolumn{5}{|l|}{$\mathrm{T}$ value } \\
\hline 1 & $74(25.9)$ & $20(27.0)$ & $54(73.0)$ & 0.731 \\
\hline $2+3+4$ & $212(74.1)$ & $53(25.0)$ & $159(75.0)$ & \\
\hline \multicolumn{5}{|l|}{ N value } \\
\hline 0 & 177 (61.9) & $48(27.1)$ & $129(72.9)$ & 0.431 \\
\hline $1+2+3$ & $109(38.1)$ & $25(22.9)$ & $84(77.1)$ & \\
\hline
\end{tabular}


(A)

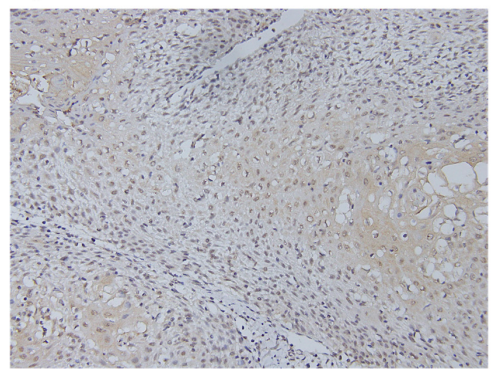

(B)

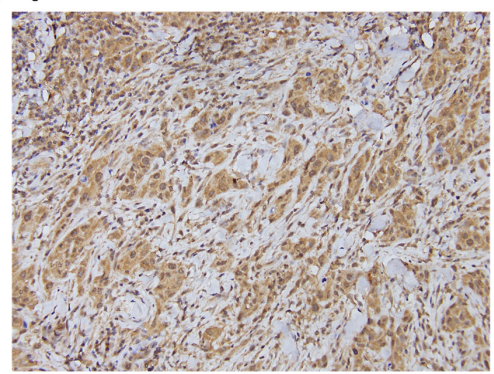

Figure 1. Representative immunostaining of KLF10 in oral squamous cell carcinoma (OSCC) specimens. The KLF10 expression levels were (A) low and (B) high.

\subsection{Prognostic Value of KLF10 Expression in Oral Cancer Tumor Specimens}

Univariate and multivariate analyses were used to evaluate the prognostic value of various parameters (Tables 2 and 3). Overall survival constituted a major measurement in both analyses. The Kaplan-Meier survival curves demonstrated a relationship between patient prognosis and KLF10 expression (Figure 2). The univariate analysis indicated that an early stage (I) of oral cancer and a high expression of KLF10 were significantly associated with a better prognosis ( $p=0.047$ and $p=0.043$, respectively). As anticipated, patients with early stage disease had a better 5 -year survival rate than those at an advanced stage (II, III, IV) $(72.2 \%$ vs. $55.2 \%$, respectively; $\log$ rank $p=0.047)$. Moreover, a higher KLF10 expression was also linked with a higher 5-year survival rate as compared to a lower expression ( $62.5 \%$ vs. $51.3 \%$, respectively; $\log \operatorname{rank} p=0.043)$. However, other parameters, such as age, gender, smoking, and betel quid chewing, had no statistically significant relationship with improved 5-year survival.

Table 2. Univariate analysis of the influence of various parameters on the overall survival of oral cancer patients.

\begin{tabular}{cccccc}
\hline & \multicolumn{5}{c}{ Overall Survival } \\
\hline Parameter & Category & 5-Year Survival (\%) & Hazard Ratio & 95\% CI & $p$ Value \\
\hline Age & $\geq 57 /<57$ & $58.8 / 58.5$ & 0.980 & $0.679-1.415$ & 0.914 \\
Gender & Male/Female & $56.8 / 69.0$ & 1.447 & $0.812-2.577$ & 0.210 \\
Smoking & Yes/No & $58.2 / 58.7$ & 0.944 & $0.651-1.368$ & 0.760 \\
Betel quid chewing & Yes/No & $60.1 / 57.9$ & 0.846 & $0.527-1.358$ & 0.489 \\
Stage & II + III + IV/I & $55.2 / 72.2$ & 1.708 & $1.007-2.896$ & 0.047 \\
KLF10 & Low /High & $51.3 / 62.5$ & 1.491 & $1.012-2.198$ & 0.043 \\
\hline
\end{tabular}

Table 3. Multivariate analysis of the influence of various parameters on the overall survival of oral cancer patients.

\begin{tabular}{cccccc}
\hline & \multicolumn{5}{c}{ Overall Survival } \\
\hline Parameter & Category & Mean Survival (Years) & HR & 95\% CI & $p$ Value \\
\hline Age & $\geq 57 /<57$ & $4.8 / 4.7$ & 0.958 & $0.656-1.400$ & 0.826 \\
Gender & Male/Female & $4.6 / 5.3$ & 1.501 & $0.817-2.757$ & 0.191 \\
Smoking & Yes/No & $4.8 / 4.7$ & 0.884 & $0.562-1.389$ & 0.592 \\
Betel quid chewing & Yes/No & $5.0 / 4.7$ & 0.855 & $0.483-1.515$ & 0.592 \\
Stage & II + III + IV/I & $4.6 / 5.5$ & 1.698 & $0.988-2.918$ & 0.055 \\
KLF10 & Low/High & $4.2 / 4.9$ & 1.528 & $1.031-2.265$ & 0.035 \\
\hline
\end{tabular}

Multivariate analysis was performed to further determine whether KLF10 expression constituted an independent prognostic marker in our selected group (Table 3). After the adjustment of the confounding factors by a linear regression model, high KLF10 expression appeared to be the only parameter significantly correlated with longer mean survival. A high expression of KLF10 was associated with longer mean survival in comparison to low expression ( 4.9 vs. 4.2 years; hazard ratio (HR): $1.528, p=0.035$ ), but other parameters, 
including early stage, were not significantly associated with better mean survival. With further included $\mathrm{T}$ value and $\mathrm{N}$ value to the multivariate analysis, the prognostic role of KLF10 remained not changed (HR: 1.716, 95\% CI: 1.154-2.551; $p=0.008$ ).

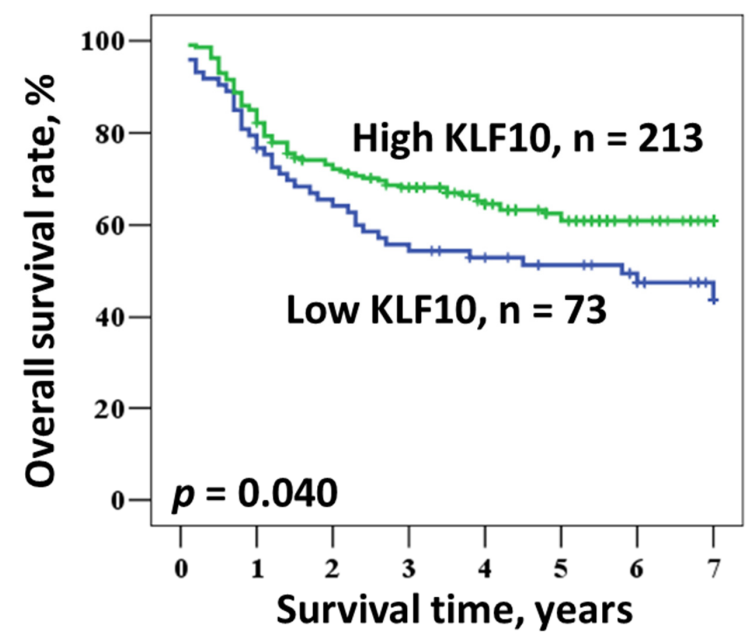

Figure 2. Kaplan-Meier survival of OSCC patients according to KLF10 expression.

\subsection{Influence of KLF10 Expression on Overall Survival According to Clinical Parameters}

A subgroup analysis was conducted using a multivariate method of determining survival outcome based on clinical parameters to more accurately identify the prognostic character of KLF10 expression (Table 4). Multivariate adjustment was performed for age, gender, smoking, betel quid, and cancer stage. Among these parameters, a significant relationship between high KLF10 expression and a higher 5-year survival rate was observed in a number of parameters, including female gender ( $88.6 \%$ vs. $44.9 \%$; HR: $7.045, p=0.016$ ), non-smokers (62.2\% vs. $49.9 \%$; HR: $1.694, p=0.04)$, cancer stage T1 $(78.9 \%$ vs. $50.0 \%$; HR: $3.074, p=0.017)$, and cancer stage N0 (73.2\% vs. $60.2 \%$; HR:1.779, $p=0.043)$.

Table 4. Multivariate analysis of the influence of KLF10 expression according to clinical parameters on overall survival in oral cancer patients.

\begin{tabular}{|c|c|c|c|c|}
\hline \multirow[b]{2}{*}{ Parameter } & \multicolumn{4}{|c|}{ Overall Survival ${ }^{1}$} \\
\hline & 5-Year Survival (\%) & HR & $95 \%$ CI & $p$ Value \\
\hline All cases & $51.3 / 62.5$ & 1.528 & $1.031-2.265$ & 0.035 \\
\hline \multicolumn{5}{|l|}{ Age (years) } \\
\hline$<57$ & $51.5 / 61.2$ & 1.435 & $0.844-2.441$ & 0.182 \\
\hline$\geq 57$ & $50.8 / 64.3$ & 1.605 & $0.869-2.965$ & 0.131 \\
\hline \multicolumn{5}{|l|}{ Gender } \\
\hline Female & $44.9 / 88.6$ & 7.045 & $1.444-34.382$ & 0.016 \\
\hline Male & $52.7 / 57.8$ & 1.289 & $0.833-1.997$ & 0.255 \\
\hline \multicolumn{5}{|l|}{ Smoke } \\
\hline Yes & $53.6 / 59.0$ & 1.262 & $0.651-2.447$ & 0.490 \\
\hline No & $49.9 / 62.2$ & 1.694 & $1.024-2.800$ & 0.040 \\
\hline \multicolumn{5}{|l|}{ Stage } \\
\hline I & $53.3 / 79.4$ & 2.588 & $0.901-7.438$ & 0.077 \\
\hline $\mathrm{II}+\mathrm{III}+\mathrm{IV}$ & $50.8 / 56.6$ & 1.395 & $0.903-2.157$ & 0.134 \\
\hline \multicolumn{5}{|l|}{$\mathrm{T}$ value } \\
\hline 1 & $50.0 / 78.9$ & 3.074 & $1.219-7.755$ & 0.017 \\
\hline $2+3+4$ & $52.0 / 54.8$ & 1.260 & $0.801-1.982$ & 0.317 \\
\hline \multicolumn{5}{|l|}{ N value } \\
\hline 0 & $60.2 / 73.2$ & 1.779 & $1.018-3.108$ & 0.043 \\
\hline $1+2+3$ & $34.1 / 41.6$ & 1.725 & $0.970-3.065$ & 0.063 \\
\hline
\end{tabular}

${ }^{1}$ Adjusted for age, gender, smoking, betel quid, and stage. 


\section{Discussion}

In our study, we enrolled 286 patients with oral cancer and analyzed the histological expression of KLF10 in specimens removed from the patients. Multivariate analysis was performed to identify the relationship between multiple factors and the 5-year survival rate. The significant character of KLF10 in predicting the clinical outcome of oral cancer was discovered. Among the patients' other documented data, a higher level of KLF10 expression under IHC staining was presented as the only factor correlating with a greater 5 -year mean survival rate. In the subgroup analysis, we found that, in the groups of female patients, non-smokers, T1 stage, and N0 stage, a high KLF10 expression significantly correlated with a greater 5-year survival rate. In the parameter of T value, a high KLF10 expression was associated with a higher 5-year survival rate in the T1 subgroup (with a tumor smaller than $2 \mathrm{~cm}$ ). On the other hand, the correlation was insignificant in the T2, 3 , and 4 groups (with a tumor exceeding $2 \mathrm{~cm}$ ). Therefore, it may be assumed that the prognostic role of KLF10 is more prominent in T1 lesions. Moreover, despite its statistical significance, the gender parameter should be interpreted cautiously because of the small sample $(n=45)$.

Several studies have broadly analyzed the probable mechanism and distinct importance of KLF10 in numerous types of cancer, such as pancreatic cancer, renal cell carcinoma, breast cancer, etc. [25-27]. However, there remained a lack of comprehensive data on the correlation between the degree of KLF10 expression and clinical prognosis, which prompted the present research to define the degree of correlation by quantifying KLF10 expression. As in previous studies, the prognostic role of biomarkers was assessed by using an immunoreactivity scoring system on pathological specimens to precisely describe the expression [22,28]. The present study, a novel model researching the relationship between high KLF10 expression and oral cancer prognosis, reproduced the scoring system to clearly address the clinical association. The majority of studies have aimed to identify the subtle character of KLF10 by discovering its sophisticated transcriptional pathway in modulating cancer progression [14-19], but few articles had investigated the direct connection between the degree of KLF10 expression and clinical outcome to elucidate the pivotal prognostic potential of KLF10 [7]. Hence, this study was designed to draw a practical clinical conclusion using 5-year survival as the primary outcome, which may aid clinicians in more precisely distinguishing a favorable prognosis from the opposite. Nevertheless, although the potential prognostic value of KLF10 was clearly demonstrated in our study, the detailed mechanism and molecular model of tumor suppression remain unclear and warrant further research.

The KLF transcription factor and another group of factors, known as transcription factor SP (specificity proteins), similarly contain three Krüppel-like zinc finger structures and are recognized as the SP/KLF family. This collective has been shown to participate in several cell functions, such as growth, apoptosis, differentiation, and angiogenesis. This illustrates that the SP/KLF family engages in multiple aspects of tumorigenesis [19]. Initially, KLF10 was identified as an early gene induced by TGF $\beta$ and was named the TGF $\beta$ inducible early gene 1 (TIEG1) $[29,30]$. The TGF $\beta$ superfamily is a group of transcription factors that was discovered to have the function of mediating fundamental cell processes, such as proliferation, differentiation, death, cytoskeletal organization, adhesion, and migration. Its transcription of target genes is controlled mainly by SMADs (SMAD family members) proteins, a collection of intracellular mediators of the TGF $\beta$ family [31]. Serving as an effector protein of TGF $\beta$-mediated cell growth control and differentiation, KLF10 is well known for its close relationship with TGF $\beta$ and consequent pivotal role in various cancers. KLF10 effectively represses cancer cell proliferation, with the overexpression of KLF10 reducing cell proliferation in many cancer types while its absence may enhance cell proliferation [19]. One previous study aimed to identify the clinical prognostic role of KLF10 in pancreatic cancer, and a higher expression of KLF10 was shown to be an independent predictor of progression-free survival and overall survival for pancreatic cancer patients [7]. 
Although the significant prognostic role of KLF10 was also identified in our study, several limitations warrant a cautious interpretation of the result. First, the pathological specimens were analyzed retrospectively after being resected from the tumor bed in a limited size, so it is possible that they were not sufficiently representative to demonstrate the protein expression of the whole tumor. Moreover, there were no data of adjacent normal tissue to compare paired tumor and normal expression. Additionally, the data were collected from patients of the same country, and the limited sample size may restrict the external validity. Moreover, a lack of information about cancer-specific death and adjuvant or neoadjuvant chemotherapy may also influence evaluation of the prognosis.

Much previous research has widely discussed the role of KLF10 in a transcriptional pathway that could potentially mediate tumorigenesis, cell proliferation, and apoptosis. Our study aimed more to directly identify the prognostic value of the biomarker and succeeded in demonstrating that a high KLF10 expression is associated with a more favorable clinical outcome in oral cancer. In light of the significant prognostic role of KLF10 shown in the present study and the few other studies that drew similar conclusions in relation to various cancers, subsequent studies are needed to develop new screening methods or therapies.

\section{Conclusions}

KLF10 expression could potentially be used as an independent prognostic marker in patients with oral cancer, especially those at the early $\mathrm{T}$ and $\mathrm{N}$ stages. However, due to the small sample size in our study, further research with larger populations is warranted to support our findings before its clinical application as a prognostic marker.

Author Contributions: Conceptualization, Y.-M.L. and W.-W.S.; data curation, Y.-J.L., P.-Y.K., C.-M.Y., and Y.-M.L.; formal analysis, Y.-J.L. and W.-W.S.; funding acquisition, W.-W.S.; investigation, C.-M.Y. and Y.-M.L.; methodology, Y.-J.L., P.-Y.K., and C.-M.Y.; supervision, W.-W.S.; validation, Y.-J.L. and C.-M.Y.; writing—original draft, P.-Y.K.; writing—review and editing, Y.-J.L., Y.-M.L., and W.-W.S. All authors have read and agreed to the published version of the manuscript.

Funding: This work was jointly supported by grants from Chung Shan Medical University Hospital research program, Taichung, Taiwan (CSH-2018-C-001).

Institutional Review Board Statement: The study was conducted according to the guidelines of the Declaration of Helsinki. The study was approved by the Institutional Review Board and Ethics Committee of the Changhua Christian Hospital, Changhua, Taiwan (IRB no. 131014) (3 March 2013).

Informed Consent Statement: The informed consent was waived as the tissues were obtained from established tissue bank.

Data Availability Statement: The data presented in this study are available on request from the corresponding author. The data are not publicly available due to possible personal information breaches though they were de-linked.

Conflicts of Interest: The authors declare no conflict of interest.

\section{References}

1. Kumar, M.; Nanavati, R.; Modi, T.; Dobariya, C. Oral cancer: Etiology and risk factors: A review. J. Cancer Res. Ther. 2016, 12, 458-463. [CrossRef] [PubMed]

2. Miranda-Filho, A.; Bray, F. Global patterns and trends in cancers of the lip, tongue and mouth. Oral Oncol. 2020, $102,104551$. [CrossRef] [PubMed]

3. Rivera, C. Essentials of oral cancer. Int. J. Clin. Exp. Pathol. 2015, 8, 11884-11894. [PubMed]

4. Siegel, R.L.; Miller, K.D.; Jemal, A. Cancer statistics, 2016. CA Cancer J. Clin. 2016, 66, 7-30. [CrossRef] [PubMed]

5. Almangush, A.; Heikkinen, I.; Mäkitie, A.A.; Coletta, R.D.; Läärä, E.; Leivo, I.; Salo, T. Prognostic biomarkers for oral tongue squamous cell carcinoma: A systematic review and meta-analysis. Br. J. Cancer 2017, 117, 856-866. [CrossRef] [PubMed]

6. Shivapurkar, N.; Reddy, J.; Chaudhary, P.M.; Gazdar, A.F. Apoptosis and lung cancer: A review. J. Cell. Biochem. 2003, 88, 885-898. [CrossRef]

7. Chang, V.H.; Chu, P.-Y.; Peng, S.-L.; Mao, T.-L.; Shan, Y.-S.; Hsu, C.-F.; Lin, C.-Y.; Tsai, K.K.; Yu, W.C.; Ch'Ang, H.-J. Krüppel-Like Factor 10 Expression as a Prognostic Indicator for Pancreatic Adenocarcinoma. Am. J. Pathol. 2012, 181, 423-430. [CrossRef]

8. Subramaniam, M.; Hawse, J.R.; Johnsen, S.A.; Spelsberg, T.C. Role of TIEG1 in biological processes and disease states. J. Cell. Biochem. 2007, 102, 539-548. [CrossRef] 
9. Weng, C.-C.; Hawse, J.R.; Subramaniam, M.; Chang, V.H.S.; Yu, W.C.Y.; Hung, W.-C.; Chen, L.-T.; Cheng, K.-H. KLF10 loss in the pancreas provokes activation of SDF-1 and induces distant metastases of pancreatic ductal adenocarcinoma in the KrasG12D p53flox/flox model. Oncogene 2017, 36, 5532-5543. [CrossRef]

10. Pearson, R.; Fleetwood, J.; Eaton, S.; Crossley, M.; Bao, S. Krüppel-like transcription factors: A functional family. Int. J. Biochem. Cell Biol. 2008, 40, 1996-2001. [CrossRef]

11. Papadakis, K.A.; Krempski, J.; Reiter, J.; Svingen, P.; Xiong, Y.; Sarmento, O.F.; Huseby, A.; Johnson, A.J.; Lomberk, G.A.; Urrutia, R.A.; et al. Krüppel-like factor KLF10 regulates transforming growth factor receptor II expression and TGF- $\beta$ signaling in CD8+ T lymphocytes. Am. J. Physiol. Cell Physiol. 2015, 308, C362-C371. [CrossRef] [PubMed]

12. Tachibana, I.; Imoto, M.; Adjei, P.N.; Gores, G.J.; Subramaniam, M.; Spelsberg, T.C.; Urrutia, R. Overexpression of the TGFbetaregulated zinc finger encoding gene, TIEG, induces apoptosis in pancreatic epithelial cells. J. Clin. Investig. 1997, 99, 2365-2374. [CrossRef] [PubMed]

13. Ellenrieder, V. TGFbeta regulated gene expression by Smads and Sp1/KLF-like transcription factors in cancer. Anticancer. Res. 2008, 28, 1531-1539. [PubMed]

14. Hsu, C.-F.; Sui, C.-L.; Wu, W.-C.; Wang, J.-J.; Yang, D.H.; Chen, Y.-C.; Yu, W.C.Y.; Chang, H.-S. Klf10 induces cell apoptosis through modulation of BI-1 expression and $\mathrm{Ca}^{2+}$ homeostasis in estrogen-responding adenocarcinoma cells. Int. J. Biochem. Cell Biol. 2011, 43, 666-673. [CrossRef]

15. Song, K.-D.; Kim, D.-J.; Lee, J.E.; Yun, C.-H.; Lee, W.-K. KLF10, transforming growth factor- $\beta$-inducible early gene 1, acts as a tumor suppressor. Biochem. Biophys. Res. Commun. 2012, 419, 388-394. [CrossRef]

16. Heo, S.-H.; Jeong, E.-S.; Lee, K.-S.; Seo, J.-H.; Lee, W.-K.; Choi, Y.-K. Krüppel-like factor 10 null mice exhibit lower tumor incidence and suppressed cellular proliferation activity following chemically induced liver tumorigenesis. Oncol. Rep. 2015, 33, 2037-2044. [CrossRef]

17. Yang, N.; Chen, J.; Zhang, H.; Wanggang, Z.; Yao, H.; Peng, Y.; Zhang, W. LncRNA OIP5-AS1 loss-induced microRNA-410 accumulation regulates cell proliferation and apoptosis by targeting KLF10 via activating PTEN/PI3K/AKT pathway in multiple myeloma. Cell Death Dis. 2017, 8, e2975. [CrossRef]

18. Marrero-Rodríguez, D.; Taniguchi-Ponciano, K.; Subramaniam, M.; Hawse, J.R.; Pitel, K.S.; La Cruz, H.A.-D.; Huerta-Padilla, V.; Ponce-Navarrete, G.; Figueroa-Corona, M.D.P.; Gomez-Virgilio, L.; et al. Krüppel-Like Factor 10 participates in cervical cancer immunoediting through transcriptional regulation of Pregnancy-Specific Beta-1 Glycoproteins. Sci. Rep. 2018, 8, 1-12. [CrossRef]

19. Memon, A.; Lee, W.-K. KLF10 as a Tumor Suppressor Gene and Its TGF- $\beta$ Signaling. Cancers 2018, 10, 161. [CrossRef]

20. Hwang, J.-C.; Sung, W.-W.; Tu, H.-P.; Hsieh, K.-C.; Yeh, C.-M.; Chen, C.-J.; Tai, H.-C.; Hsu, C.-T.; Shieh, G.S.; Chang, J.-G.; et al. The Overexpression of FEN1 and RAD54B May Act as Independent Prognostic Factors of Lung Adenocarcinoma. PLoS ONE 2015, 10, e0139435. [CrossRef]

21. Lee, Y.-J.; Chan, L.; Yeh, C.-M.; Lee, C.-H.; Sung, W.-W. Overexpression of KLF17 Predicts a Favorable Prognosis in Patients with Oral Squamous Cell Carcinoma: A Retrospective Study. Medicine 2020, 56, 57. [CrossRef] [PubMed]

22. Sung, W.-W.; Lin, Y.-M.; Wu, P.-R.; Yen, H.; Lai, H.-W.; Su, T.-C.; Huang, R.-H.; Wen, C.-K.; Chen, C.-Y.; Chen, C.-J.; et al. High nuclear/cytoplasmic ratio of Cdk1 expression predicts poor prognosis in colorectal cancer patients. BMC Cancer 2014, 14, 951. [CrossRef] [PubMed]

23. Sung, W.-W.; Wang, Y.-C.; Cheng, Y.-W.; Lee, M.-C.; Yeh, K.-T.; Wang, L.; Wang, J.; Chen, C.-Y.; Lee, H. A Polymorphic -844T/C inFasLPromoter Predicts Survival and Relapse in Non-Small Cell Lung Cancer. Clin. Cancer Res. 2011, 17, 5991-5999. [CrossRef] [PubMed]

24. Sung, W.-W.; Wang, Y.-C.; Lin, P.-L.; Cheng, Y.-W.; Chen, C.-Y.; Wu, T.-C.; Lee, H. IL-10 Promotes Tumor Aggressiveness via Upregulation of CIP2A Transcription in Lung Adenocarcinoma. Clin. Cancer Res. 2013, 19, 4092-4103. [CrossRef] [PubMed]

25. Ivanov, S.V.; Ivanova, A.V.; Salnikow, K.; Timofeeva, O.; Subramaniam, M.; Lerman, M.I. Two novel VHL targets, TGFBI (BIGH3) and its transactivator KLF10, are up-regulated in renal clear cell carcinoma and other tumors. Biochem. Biophys. Res. Commun. 2008, 370, 536-540. [CrossRef]

26. Jiang, L.; Wang, F.; Lin, F.; Gao, S.; Tan, Y.; Han, Y.; Chen, C.; Wu, J. Lentivirus-mediated overexpression of TGF- $\beta$ inducible early gene 1 inhibits SW1990 pancreatic cancer cell growth. Cell Biol. Int. 2011, 35, 891-896. [CrossRef]

27. Reinholz, M.; An, M.-W.; Johnsen, S.A.; Subramaniam, M.; Suman, V.J.; Ingle, J.N.; Roche, P.C.; Spelsberg, T.C. Differential Gene Expression of TGF $\beta$ Inducible Early Gene (TIEG), Smad7, Smad2 and Bard1 in Normal and Malignant Breast Tissue. Breast Cancer Res. Treat. 2004, 86, 75-88. [CrossRef]

28. Su, T.-C.; Chen, C.-Y.; Tsai, W.-C.; Hsu, H.-T.; Yen, H.-H.; Sung, W.-W.; Chen, C.-J. Cytoplasmic, nuclear, and total PBK/TOPK expression is associated with prognosis in colorectal cancer patients: A retrospective analysis based on immunohistochemistry stain of tissue microarrays. PLoS ONE 2018, 13, e0204866. [CrossRef]

29. Anderson, K.P.; Kern, C.B.; Crable, S.C.; Lingrel, J.B. Isolation of a gene encoding a functional zinc finger protein homologous to erythroid Kruppel-like factor: Identification of a new multigene family. Mol. Cell Biol. 1995, 15, 5957-5965. [CrossRef]

30. Subramaniam, M.; Harris, S.A.; Oursler, M.J.; Rasmussen, K.; Riggs, B.L.; Spelsberg, T.C. Identification of a novel TGF-betaregulated gene encoding a putative zinc finger protein in human osteoblasts. Nucleic Acids Res. 1995, 23, 4907-4912. [CrossRef]

31. Wrighton, K.H.; Lin, X.; Feng, X.H. Phospho-control of TGF-beta superfamily signaling. Cell Res. 2009, 19, 8-20. [CrossRef] [PubMed] 